\title{
Back to the roots?
}

\section{"Interkulturelle Psychotherapie» \\ und das Jung'sche Archetypenkonzept in der (Post-)Migrationsgesellschaft}

\author{
Inga Oberzaucher-Tölke
}

Journal für Psychologie, 26(2), 184-203

https://doi.org/10.30820/8248.10

www.journal-fuer-psychologie.de

\section{Zusammenfassung}

Die Herausforderungen an Psychotherapie in der deutschen Migrationsgesellschaft werden nicht erst seit den aktuellen Migrations- und Fluchtbewegungen viel diskutiert. Für die Psychoanalyse stellt sich dabei unter anderem die Frage, welchen Nutzen klassische analytische Konzepte noch (oder wieder?) für eine Psychotherapie in der sich verändernden Migrationsgesellschaft haben. Diese müssen jedoch immer auch vor dem Hintergrund ihres gesellschaftlichen Entstehungskontextes analysiert und bewertet werden. Im folgenden Artikel wird deshalb das Archetypenkonzept Carl Gustav Jungs, das in seiner universellen Ausrichtung für eine Psychotherapie in der Migrationsgesellschaft zunächst vielversprechend erscheint, aus einer interdisziplinären, rassismus- und diskurskritischen Perspektive diskutiert und schließlich seine postmigrantischen Potenziale herausgearbeitet.

Schlüsselwörter: (Post-)Migration, Diskurs, Rassismus, Kultur, Psychotherapie, Psychoanalyse, Archetypenlehre

\section{Summary}

Back to the roots? »Intercultural Psychotherapy« and the Jungian Archetype Model in (Post-)Migration Society

In the German migration society, psychotherapy has been debated and challenged not only since recent migration movements. Concerning psychoanalysis it seems significant to question the benefit of classical analytical concepts for psychotherapy in changing migration society. At the same time these concepts have to be reviewed by particularly taking into account their societal genesis. Initially, the archetype model by Carl Gustav Jung in its universal orientation appears promising for psychotherapy in the German migration society. Nonetheless in this article the concept is reviewed from an interdisciplinary discourse and racism critical perspective, before illustrating its potential for a contemporary psychotherapy referring to $\gg$ post-migration $\ll$. 
Keywords: (post-)migration, discourse, racism, culture, psychotherapy, psychoanalysis, archetype model

\section{Einleitung}

Migrationsbewegungen sind sowohl für Europa als auch für Deutschland seit jeher von zentraler Bedeutung (Meinhardt \& Schulz-Kaempf, 2015; Yildiz \& Hill, 2015). Trotz der aktuellen Tendenz einer verschärften Migrations- und vor allem Asylpolitik kommen Phänomenen kultureller, lingualer, juristischer und (geo-)politischer Grenzüberschreitungen tagtäglich große Bedeutung zu (Mecheril et al., 2013). Erfahrungen von (An-)Kommen, Gehen und Bleiben sind damit für unsere Gesellschaft konstitutiv und die damit verbundenen Themen von Abschied, Übergang, Fremdheit und Heimat nicht zuletzt auch für die Psychotherapie relevant. Der Alltäglichkeit von Migration und der damit verbundenen Entstehung von Zwischenwelten und hybriden Identitäten stehen jedoch (Alltags-)Rassismen und Fremdheitskonstruktionen gegenüber, die Migration und ihre Folgen als »Problem « oder zumindest »Sonderfall « erscheinen lassen. Hierzu tragen auch psychotherapeutische Fachdiskurse bei, die Therapie mit sogenannten »Patient_innen mit Migrationshintergrund « meist unter dem Stichwort »Interkulturelle Psychotherapie « verhandeln. Wenn überhaupt nur marginal thematisiert wird hingegen, dass Migration und ihre Folgen die gesamte Gesellschaft betreffen, also in der Psychotherapie neben den »Patient_innen mit Migrationshintergrund « auch die (oft) mehrheitsangehörigen Therapeut_innen. Dies lässt sich sowohl mit der intersubjektiven Wende in der Psychoanalyse (Erman, 2014) als auch aus rassismuskritischer Perspektive (Mecheril, 2004) begründen: Patient_in und Therapeut_in müssen demnach immer auch als in gesellschaftliche Verhältnisse eingebunden verstanden werden, welche die Subjektformen »deutsch « und »nicht-deutsch «, aber auch »Patient_in « und »Therapeut_in « erst hervorbringen.

Im Kontext von Migration zeigen sich im psychotherapeutischen Fachdiskurs Analogien zu Fachdiskursen der Pädagogik, hier entwickelte sich bereits in den 1980er Jahren aus der »Ausländerpädagogik « eine sogenannte »Interkulturelle Pädagogik «. Auch diese Diskurse machten zunächst »Ausländer_innen «, dann »Migrant_innen « und ihre »Kulturen « zu ihrem Gegenstand. Kritik an der Interkulturellen Pädagogik und dem sie konstituierenden Kulturbegriff führte zu einem erneuten Paradigmenwechsel, aus dem sich schließlich die Perspektive »Rassismuskritik « entwickelte. Diese hat den Anspruch, statt der »Migrant_innen « und »kulturell Anderen « die Gesellschaft als Ganzes und ihre Machtverhältnisse in den Fokus zu nehmen. Ein vergleichbarer Paradigmenwechsel ist in der Psychotherapie, mit Ausnahme einiger ethnopsychoanalytisch informierter Ansätze, bisher ausgeblieben, wie der rassismuskritische Blick auf 
Diskurse Interkultureller Psychotherapie zeigt (siehe 2. sowie ausführlich OberzaucherTölke, 2018, 2017). Die Zusammenführung beider Disziplinen im Rahmen dieses Artikels begründet sich weiterhin durch die Gemeinsamkeit der Arbeit am Menschen mit einer je spezifischen asymmetrischen Konstellation zwischen Professionellen und ihrer Zielgruppe (z.B. Therapeut_in-Patient_in, Lehrer_in-Schüler_in).

Im Folgenden werden die Entwicklungen von der Interkulturellen zur Rassismuskritischen Pädagogik zunächst genauer nachgezeichnet (1.), um schließlich auf ein exemplarisches Textbeispiel des Diskurses der Interkulturellen Psychotherapie übertragen zu werden (2.). Zur Beobachtung und Analyse aktueller empirischer Realitäten in der Migrationsgesellschaft etablieren sich derzeit neben der pädagogischen Rassismuskritik » postmigrantische « Perspektiven in den Kultur- und Sozialwissenschaften. Diese werden gewissermaßen als Fortführung bzw. empirische Ergänzung der Rassismuskritik skizziert und daraus alternative Sichtweisen auf Migrationsgesellschaft und Psychotherapie jenseits der Interkulturalität entwickelt (3.). Schließlich wird mit dem Archetypenkonzept Carl Gustav Jungs ein psychoanalytisches Konzept in den Blick genommen, das für eine postmigrantische Psychotherapie zunächst vielversprechend erscheint, weist es doch im »Erkennen wesentlicher Merkmale des menschlichen Daseins über ethnische, kulturelle und auch soziale Schranken hinaus « (Gekeler, 2012, S. 358). Aus den zuvor entwickelten diskursanalytischen und rassismuskritischen Perspektiven wird das Konzept jedoch zunächst einer kritischen Revision unterzogen und dann seine Potenziale für eine postmigrantisch ausgerichtete Psychotherapie herausgearbeitet (4.).

\section{Diskurse Interkultureller Pädagogik und die rassismuskritische Perspektive}

Im Folgenden wird aufgezeigt, wie in der Pädagogik in den letzten Jahrzehnten konzeptionell mit Migration umgegangen wurde, um sich vor diesem Hintergrund dann den einschlägigen Diskursen der Psychotherapie anzunähern. Es wird dabei eine Diskursperspektive auf Pädagogik und Migration eingenommen, es geht also nicht vorrangig um den pädagogisch-praktischen Umgang mit Migration oder gar Migrant_innen. Vielmehr wird rezipiert, wie über Migration gesprochen wird und warum in diesem Zusammenhang zunächst »Ausländer «, dann »Migrant_innen « oder »Menschen aus anderen Kulturen « zum Thema gemacht wurden und werden, die sogenannte Mehrheitsgesellschaft jedoch nur in Ausnahmefällen besprochen wird. Migration wird als Gegenstand von Diskursen und als Gegenstand politischer und alltagsweltlicher Auseinandersetzungen verstanden, in denen die Frage, ob es eher um gesellschaftlichen Erhalt oder Umgestaltung geht, zum Thema wird (Broden \& Mecheril, 2007). Diskur- 
se lassen sich in diesem Zusammenhang » als mehr oder weniger erfolgreiche Versuche verstehen, Bedeutungszuschreibungen und Sinn-Ordnungen zumindest aufZeit zu stabilisieren und dadurch eine kollektiv verbindliche Wissensordnung in einem sozialen Ensemble zu institutionalisieren « (Keller, 2011, S. 7). Eine solche Bedeutungszuschreibung wäre z.B. das Label »Migrant_in « an bestimmte Mitglieder einer Gesellschaft. Die dadurch entstehende Wissensordnung wäre dann z. B. das Wissen darüber, wer ein_e »Migrant_in « ist und wer nicht, diese Wissensordnung wiederum wird unter anderem durch offizielle Definitionen (z. B. des »Migrationshintergrunds « durch das Statistische Bundesamt; www.destatis.de) institutionalisiert. Auch pädagogische und psychotherapeutische Konzeptionen zum Umgang mit »Migrant_innen «, die im Rahmen wissenschaftlicher Publikationen formuliert und verbreitet werden, tragen zur Institutionalisierung dieser Wissensordnung bei. Mit Michel Foucault, einem der Begründer der Diskurstheorie, sei hinzugefügt, dass Diskurse grundsätzlich die Dinge bilden, über die sie sprechen, und es damit keine vordiskursive Wirklichkeit gibt (z. B. Bublitz, 2003) - das heißt, der Diskurs über »Migrant_innen« macht die spezifische Subjektform »Migrant_in « und die an sie vorgenommenen Zuschreibungen erst möglich und hat damit grundsätzlich eine konstituierende Wirkung.

Aus dieser Perspektive wird deutlich, dass der pädagogische Umgang mit Migration zu unterschiedlichen Zeiten unterschiedlichen Diskursen unterlag und unterliegt: Die Erziehungswissenschaftler_innen Isabell Diehm und Frank-Olaf Radtke (1999) zeigen auf, dass die Entwicklung der Ausländerpädagogik zunächst im Zusammenhang mit der Gastarbeiter_innenmigration nach Deutschland zu sehen ist. Als Kritik und in Abgrenzung von dieser eher defizitorientierten Programmatik entwickelte sich in den 1980er Jahren die Interkulturelle Pädagogik, welche »als pädagogisches Programm nicht nur für die Schule, sondern als durchgängiges Erziehungsprinzip auch für alle vor-, außer- und nachschulischen Einrichtungen zu begründen und zu implementieren « (ebd., S. 127) versucht wurde. In den 1990er Jahren fanden nun wiederum (diskursive) Abgrenzungsversuche von der Interkulturellen Pädagogik statt, wobei die Kritik vor allem unter den Stichworten Ethnisierung/Kulturalisierung und Pädagogisierung/Curricularisierung zusammenzufassen ist (ebd., S. 146ff.). Im Folgenden soll es vor allem um die Kritik an der Kulturalisierung gehen, da diese meines Erachtens auch für die Interkulturelle Psychotherapie besonders relevant ist. Nach Diehm und Radtke meint Kulturalisierung eine »Verengung, Vereinseitigung oder Überbetonung der Beschreibung sozialer Wirklichkeit/Probleme mit der Unterscheidung >Kultur $<\ll$ (ebd., S. 147). Der Begriff »Kultur « wird in der Interkulturellen Pädagogik als (ausschließliche) Unterscheidungskategorie genutzt, um Schüler_innen und ihr Lern- und Sozialverhalten zu kategorisieren. Damit erscheinen Schüler_innen als »kleine Repräsentanten >ihrer Kultur « (ebd.), was auch als »ethnisch-kulturelle Zwangsjacke « verstanden werden kann (z.B. Hess, 2015). Weitere Problematiken von Kulturalisie- 
rung nicht nur in der Pädagogik sind der Aspekt des Otherings durch ihre Verwendung (Fremdmachung von » kulturell Anderen « zur Stabilisierung eines homogenen Selbstbildes), die häufige Verkürzung des Begriffs »Kultur « im Sinne einer Nationalkultur (z. B. die türkische, die italienische, die deutsche Kultur), die hierarchische Bewertung von $\gg$ Kulturen « (z. B. als modern vs. rückständig) sowie (ungleiche) gesellschaftliche Machtverhältnisse, in denen diese vorgenommen werden (Oberzaucher-Tölke, 2013).

Unter anderem aus dieser Kritik an Kulturalisierungen der Interkulturellen Pädagogik sowie vor dem Hintergrund (sozial-)konstruktivistischer, rassismus- und machttheoretischer Erkenntnistheorien entwickelte sich in den 2000er Jahren die Perspektive der Rassismuskritischen Migrationspädagogik, die der Psychologe und Erziehungswissenschaftler Paul Mecheril (2004) als »reflexive Schule « bezeichnet. Hier wird das Augenmerk nicht mehr auf unterschiedliche »Kulturen « gelegt, sondern darauf, wie eine Unterscheidung in »eigene « und »fremde Kultur « überhaupt erst möglich wird und wie und warum sie eine solche (diskursive) Wirkkraft erlangt, dass sie über Zugehörigkeit und Nicht-Zugehörigkeit entscheidet. Die Rassismuskritische Migrationspädagogik begründet dies mit einer grundsätzlich rassistisch strukturierten Gesellschaftsordnung. Rassismus wird dabei nicht als Einstellung oder Haltung verstanden, die individuell überwunden werden muss, sondern als machtvolles, mit Rasseoder Kulturkonstruktionen operierendes System von gesellschaftlichen Diskursen und Praxen, mit denen Ungleichbehandlung und hegemoniale Machtverhältnisse wirksam und plausibilisiert werden (Mecheril \& Melter, 2009). Das Differenzierungsschema zwischen »Migrant_innen « und »Nicht-Migrant_innen « wird damit in einen allgemeinen Zusammenhang gesellschaftlich dominanter Unterscheidungslogiken gestellt. Denn nur vor diesem Hintergrund, so Mecheril (2004, S. 190), seien ethnisch-kulturelle Unterscheidungskategorien möglich und wirkmächtig, z. B. als Selektionskriterium in der Schule sowie als Begründungs- und Legitimationsmuster etwa für das Verhalten von Schüler_innen. Meist unter Rückgriff auf die » eigene « und die »fremde Kultur « steht damit die »binäre Unterscheidung zwischen Wir und Nicht-Wir « (ebd., S. 187) im Zentrum des rassistischen Systems, das aus kollektiven Bildern, Erzählungen/Diskursen und gesellschaftlichen Institutionen besteht und damit historische und aktuelle Machtverhältnisse legitimiert und reproduziert (ebd.). Vor diesem Hintergrund benennt Mecheril die Dekonstruktion binärer Schemata, z. B. der Unterscheidung in ein »natio-ethno-kulturelles Wir « und »Nicht-Wir «1, als einen der Grundzüge einer Rassismuskritischen Migrationspädagogik. Bei der Dekonstruktion binärer Schemata handelt es sich um eine Interpretationsperspektive, die »dazu beiträgt, Texte und Begriffe auf die sie ermöglichenden binären Schemata und ausschließenden Dualismen zu betrachten « (ebd., S. 210). Diese Perspektive soll im Folgenden auf ein Textbeispiel des psychotherapeutischen Diskurses zur Therapie von »Migrant_innen « angewandt und gleichsam illustriert werden. 


\section{Diskurse Interkultureller Psychotherapie aus rassismuskritischer Perspektive}

Auch in Psychotherapie und Psychiatrie haben sich in den letzten Jahrzehnten verschiedene Modelle und Konzepte »Inter- und Transkultureller Psychotherapie « für die Arbeit mit »Migrant_innen « entwickelt, auf die in einschlägigen Fachpublikationen Bezug genommen wird bzw. die dort konzeptionell umrissen werden (z. B. Hegemann \& Salman, 2010; Machleidt \& Heinz, 2011; Wohlfahrt \& Zaumseil, 2006). Im Rahmen dieses Spezialdiskurses (Jäger, 1993) wird Psychotherapie in der Migrationsgesellschaft verhandelt und, so die These nach Michel Foucault, der »kulturell andere Patient « als ihr Gegenstand hergestellt (Oberzaucher-Tölke, 2014). Eine ausführliche Analyse des Diskurses hat weiterhin gezeigt, dass kultureller Rassismus und diskursive Praxen von Othering als kontinuierliche Phänomene desselben zu verstehen sind (Oberzaucher-Tölke, 2018): »Kultur « erscheint größtenteils als unveränderliche, wesenhafte Erscheinung von Menschen und muss damit als ein Äquivalent zu Rassismus zugrunde liegenden Rassekonstruktionen verstanden werden. Gleichzeitig werden bestimmte, über Herkunft und Kultur definierte Gruppen zu »Fremden « gemacht, worüber gleichzeitig ein »Wir « konstruiert wird, das unhinterfragt und vermeintlich homogen bleibt (ebd.). Im Folgenden soll nun anhand eines ausgewählten Diskursfragments exemplarisch aufgezeigt werden, wie im psychotherapeutischen Diskurs im Hinblick auf Migration und kulturelle Differenz auf binäre, homogenisierende und hierarchisierende Unterscheidungslogiken zurückgegriffen wird, die in Analogie zu rassistischen Logiken stehen.

Ahmet Kimil et al. (2013) vom Ethno-Medizinischen Zentrum Hannover schreiben in einem Text über »Ressourcen und Schwierigkeiten von MigrantInnen und ihren Familien im Kontext von Beratung und Therapie « von Migrant_innen mit speziellen Bedürfnissen und Besonderheiten, auf die Berater_innen, Therapeut_innen und Organisationen mittels Konzepten und Angeboten eingehen müssten (ebd., S. 63). Dies wird dadurch begründet, dass »MigrantInnen auf andere, migrationsgeprägte kulturelle Muster, insbesondere andere Familienbilder, zurückgreifen als die Mehrheitsbevölkerung « (ebd.). »Migrant_innen « werden hier also als eine besondere, homogene Patient_innengruppe von Therapie und Beratung konstruiert, die durch ihre kulturelle Prägung » anders « seien als die Mehrheitsgesellschaft und damit eine binäre, natioethno-kulturelle Unterscheidung in »Mehrheitsbevölkerung « und »Migrant_innen « vorgenommen. Die angenommene Differenz der »kulturellen Muster « der »MigrantInnen« wird später im Text als ein »Wandel der Wir-Ich-Balancen« (ebd., S. 64) konkretisiert. Dieser Wandel führe, so die Autoren, dazu, dass die »>entwickelteren< westlichen Gesellschaften « ihren Akzent bei der »Verhaltenssteuerung « stärker auf den $\gg$ Ich-Pol « legten, die »traditionellen Kulturen « hingegen stärker auf den »WirPol $\ll$ (ebd.). Der Konstruktion kulturell anderer Patient_innen als »Migrant_innen « 
und »Angehörige traditioneller Kulturen « wird also »die Mehrheitsbevölkerung « oder »die Einheimischen « (ebd., S. 66) als Angehörige »moderner Kulturen« gegenübergestellt. Beide Gruppen werden dabei sowohl als homogen als auch in ihrer Homogenität als different oder sogar gegensätzlich dargestellt. Gleichzeitig werden ihnen pauschal eine »traditionelle « und eine »moderne « Kultur zugeschrieben, was zusätzlich zur Konstruktion des Andersseins eine implizite Bewertung von zeitgemäß und nicht-zeitgemäß, modern und zurückgeblieben enthält.

In Anschluss an dieses Beispiel wird nun mit der postmigrantischen Perspektive eine Perspektive auf Migration vorgestellt, die von ihren Vertreter_innen als $\gg$ innovativer Bruch « bezeichnet wird, der »Dualismen von westlich/nichtwestlich, Inländer/Ausländer, die bisher als Wegweiser der gesellschaftlichen Wahrnehmung fungierten, radikal infrage [stellt] « (Yildiz, 2015, S. 21). Stattdessen rückt die Komplexität und vor allem die Hybridität des Lebens der Migrationsgesellschaft in den Fokus und wird gleichsam anerkannt - was weitreichende Konsequenzen auch für eine sich in ihr verortende Psychotherapie hat.

\section{4. "Wege aus dem Rassismus? «² - Postmigrantische Perspektiven auf Gesellschaft}

»[N]ichts widerspricht dem Rassismus so sehr wie die Mischung « (Mecheril, 2004, S. 212), und damit birgt die praktische Anerkennung von Mehrfachzugehörigkeit und die Anerkennung hybrider Phänomene ein bedeutsames widerständiges Potenzial gegenüber Rassismus (ebd.). Eine solche Anerkennung nimmt die Perspektive des Postmigrantischen vor: Der Begriff »postmigrantisch « tauchte in der Wissenschaft zum ersten Mal 1995 im englischsprachigen Raum auf (Yildiz, 2015). In Deutschland wurde er im Kontext postmigrantischen Theaters von der Berlinerin Shermin Langhoff in Umlauf gebracht, die mit dem Begriff »die Praxis [kritisiert], auch Menschen ohne jegliche eigene Migrationserfahrung als Migrantinnen zu bezeichnen [und] somit Migration als Sonderstatus über Generationen hinweg festzuschreiben « (Römhild, 2014, S. 256). Angelehnt ist der Begriff weiterhin an die Postkoloniale Theorie, die die Geschichtsschreibung des Kolonialismus von westlicher Hegemonie befreien möchte und stattdessen Kolonialgeschichte »aus der Erfahrung und Perspektive der Kolonialisierten erzählt« (Yildiz, 2015, S. 19). Die Postcolonial Studies (zur Einführung siehe Castro Varela \& Dhawan, 2015; Kerner, 2012) untersuchen so unter anderem die Kontinuitäten kolonialer Machtverhältnisse und Identitäten, die im europäischen Selbstverständnis offiziell als überwunden dargestellt werden.

Die Ethnologin Regina Römhild (2015) postuliert, dass wir längst in einer postmigrantischen Gesellschaft lebten, »die überall und dauerhaft von den Erfahrungen und 
Wirkungen des Kommens, Gehens und Bleibens geprägt ist « (ebd., S. 37). Dennoch werde weiterhin von »Einwanderung « und »Integration « gesprochen, als hätte die Mehrheitsgesellschaft mit Migration nichts zu tun. Über den Schlüsselbegriff $\gg$ Kultur « und die darüber zugeschriebene Fremdheit würden Migrant_innen und ihre Kinder und Enkel immer wieder zu den problematischen »Anderen « der eigenen Gesellschaft gemacht (ebd.). Der neue Blick des Postmigrantischen, der nach dem Soziologen und Erziehungswissenschaftler Erol Yildiz (2015) den »Aufbruch in eine neue Geschichtlichkeit « einläutet, soll hingegen durch die Perspektiven derer, die Migrationsprozesse direkt oder indirekt erlebt haben, die »Geschichte der Migration neu [...] erzählen und das gesamte Feld der Migration radikal neu [...] denken« (ebd., S. 21). Damit rücken statt integrativer Leistungen von Migrant_innen in die Mehrheitsgesellschaft »Prozesse von Entortung und Neuverortung, Mehrdeutigkeit und Grenzbiografien ins Blickfeld « (ebd.). Diese Perspektive des Dazwischen kann als eine dritte, hybride Perspektive zwischen $\gg$ Migrant « und $\gg$ Nicht-Migrant $\ll, ~ \gg$ deutsch « und $\gg$ nichtdeutsch «, »Eigen-« und »Fremdkultur « betrachtet werden. Gleichsam hat sie, im Sinne einer Graswurzelbewegung, ihren Nährboden in den neuen, hybriden Lebenswirklichkeiten der Migrationsgesellschaft.

Als eine wesentliche Dimension der postmigrantischen Perspektive nennt Yildiz

»die Nachfolgegeneration der Gastarbeiter, die nicht über die gleichen Migrationserfahrungen verfügen wie ihre Eltern oder Großeltern. Sie sind in Köln, Berlin oder Wien aufgewachsen und haben hier ihre Sozialisationsprozesse durchlaufen. Dennoch werden sie von außen als >Migranten < wahrgenommen. In diesem Kontext taucht auch der Begriff $>$ Migrationshintergrund < auf, der seit einigen Jahren als die politisch korrekte Bezeichnung verhandelt wird. In der Auseinandersetzung mit der offiziellen Benennungspraxis entwickeln die betreffenden Generationen unterschiedliche Gegenstrategien und Selbstbezeichnungen, die oft irritierend auf etablierte Wahrnehmungen wirken: >Kanak Attack<, $>$ Tschuschenpower $<$, $>$ Migrantenstadl $<$, $>$ die Unmündigen $<$ usw. Die unterschiedlichen Verortungspraktiken, die aus dieser Auseinandersetzung sichtbar werden bzw. hervorgehen, sind $[\ldots]$ als $>$ postmigrantisch $<$ zu verzeichnen $\ll($ ebd., S. 22).

Das Postmigrantische funktioniert damit als »Analysekategorie für eine soziale Situation von Mobilität und Diversität « (ebd.), die Brüche, Mehrdeutigkeiten und Hybriditäten sichtbar macht. Sie versteht sich als $\gg$ Kampfbegriff gegen $>$ Migrantisierung< und Marginalisierung von Menschen, die sich als integraler Bestandteil der Gesellschaft sehen « (ebd.), und weist einen explizit widerständigen Charakter gegenüber herrschenden Diskursen auf.

Grundsätzlich ist zu überlegen, ob aufgrund der heutigen Gesellschaftsstruktur Deutschlands die Begriffe der Mehrheitsgesellschaft und ihrer Minderheiten nicht oh- 
nehin bereits ad absurdum geführt wurde. Römhild zeigt hierzu anhand der Stadt Frankfurt am Main auf, was sicherlich auch auf andere deutsche Städte zu übertragen ist:

$\gg[\mathrm{H}]$ ier haben heute schon $60 \%$ der Kinder und Jugendlichen einen so genannten Migrationshintergrund, d.h. sie sind mit ihren Eltern aus dem Ausland nach Frankfurt gezogen oder als sogenannte zweite oder dritte Generation von Einwander*innen in der Stadt geboren. Diese Zahl stellt nicht nur die übliche Vorstellung von Mehrheit und Minderheiten auf den Kopf, sie widerspricht auch dem überstrapaziertem Bild der Migrant*innen als sozialer und kultureller Problemgruppe. Stattdessen kommen diese Kinder und Jugendlichen aus höchst unterschiedlichen Verhältnissen, sie wohnen in praktisch allen Stadtteilen und ihre Eltern sind in allen Domänen der städtischen Ökonomie beschäftigt: von den oberen Büroetagen in den Hochhaustürmen der globalisierten Dienstleistungsunternehmen über ein breites Mittelfeld bürgerlicher Berufe und selbstständiger Geschäftsleute bis zu den Schluchten irregulärer, prekärer Tätigkeiten und illegalisierter Existenzen « (ebd., S. 42).

Schauen wir nun aus dieser Perspektive auf (Fach-)Diskurse zu Psychotherapie und Migration, so werden auch diese in gewisser Weise ad absurdum geführt. Dass eine spezifische Psychotherapie für »Migrant_innen « diskutiert wird, erscheint vor diesem Hintergrund als nicht zeitgemäß und an längst überholten binären Unterscheidungen zwischen »Migrant_in « und »Nicht-Migrant_in « orientiert. Die Sozialwissenschaftlerin Naika Foroutan (2015) spricht in diesem Zusammenhang von der postmigrantischen Gesellschaft als »Aushandlungsgesellschaft «, in der bisherige Konzepte nicht mehr greifen und vieles neu ausgehandelt werden muss. Nach Foroutan müssten sich die bisher kulturell, ethnisch und religiös Etablierten erst an diese Aushandlung von Positionen, Zugängen, Ressourcen und Normen gewöhnen (ebd.). Sich als mehrheitsangehörige_r Therapeut_in als integraler Teil einer postmigrantischen Gesellschaft zu verstehen, bedeutet also eine Adaption des Selbstbildes und des eigenen Verständnisses von Gesellschaft und Psychotherapie. Damit gilt es zunächst, auch eigene Migrationserfahrungen von Kommen, Gehen und Bleiben in den Blick zu nehmen, sowie die eigenen Erfahrungen von Zugehörigkeit und Nicht-Zugehörigkeit, Fremdheit und Vertrautheit sowie von Hybridität zu reflektieren. Schließlich geht es im Rahmen einer Critical Whiteness darum, sich der eigenen Positionierung in gesellschaftlichen Machtverhältnissen und daraus resultierender Selbstverständnisse, Empfindungen und (therapeutischer) Deutungen bewusst zu werden (hierzu ausführlich Oberzaucher-Tölke, 2017).

Vor diesem Hintergrund sollen nun Aspekte der (etablierten) Archetypenlehre Carl Gustav Jungs im Sinne einer postmigrantischen Revision kritisch betrachtet und schließlich ihre Potenziale für eine postmigrantische Psychotherapie herausgearbeitet werden. 


\section{Archetypenlehre revisited - Potenziale für eine postmigrantische Psychotherapie}

Am Beispiel der Archetypenlehre nach Carl Gustav Jung, dem Begründer der Analytischen Psychologie, soll nun veranschaulicht werden, welche Bedeutungen die zuvor eingeführten diskurstheoretischen, rassismuskritischen und postmigrantischen Perspektiven für die Psychotherapie haben können. Nach einer Einführung in das Konzept der Archetypen wird es zunächst einer kritischen Revision unterzogen und schließlich ihr postmigrantisches Potenzial herausgearbeitet.

\subsection{Archetypenlehre nach C. G. Jung}

Das Archetypenkonzept ist nach dem Psychologen und Psychoanalytiker Christian Roesler (2009) eines der bekanntesten und zugleich charakteristischsten Konzepte der Jung'schen Psychologie, die sich hier am deutlichsten von anderen psychologischen Theorien unterscheidet (ebd., S. 267). Die Archetypen werden von C. G. Jung im Bereich des kollektiven Unbewussten verortet. Bereits mit der Annahme der Existenz desselben wendete er sich deutlich von der klassischen psychoanalytischen Lehre Sigmund Freuds ab, sodass das kollektive Unbewusste als »Kernstück « der von Jung begründeten Analytischen Psychologie betrachtet werden kann (Schnocks, 2013, S. 42). Das kollektive Unbewusste ist nach C. G. Jung vom persönlichen Unbewussten dadurch zu unterscheiden, dass es » seine Existenz nicht persönlicher Erfahrung verdankt und daher keine persönliche Erwerbung ist« (Jung, 1995 [1936a], §88), sondern seine Inhalte ausschließlich vererbbar seien (ebd.). Das kollektive Unbewusste bestehe » aus präexistenten Formen, Archetypen, die erst sekundär bewusst werden können und den Inhalten des Bewusstseins festumrissene Form verleihen « (ebd., §90), die Archetypen stellten damit das Grundmuster instinktiven Verhaltens dar (ebd., \$1). Eine Form dieser Bewusstwerdung sind Personifizierungen der Archetypen (z. B. der Schatten, die Anima und der alte Weise; vgl. Jung, 1995 [1934]), die aus phänomenologischer Perspektive als Bilder der Seele zu sehen sind. Trotz ihres archetypischen Ursprungs müssen diese Bilder, z. B. im Rahmen von Psychotherapie, auf ihre individuelle persönliche Bedeutung hin untersucht werden (Vogel, 2012). Damit haben sie den Status von Symbolen, die »vieldeutig, ahnungsreich und im letzten Grund unausschöpfbar« (Jung, 1995 [1934], §80) sind. Archetypen sind also vom Einzelnen ausschließlich symbolisch erlebbar und ihre präexistenten Formen damit individuell auszufüllen.

Gleichzeitig können die durch Archetypen hervorgebrachten Bilder als gemeinsame, kollektive Menschheitserfahrungen gelten, da »vor allem die elementarsten mensch- 
lichen Erfahrungen wie Geburt, Ehe, Mutterschaft, Trennung, Tod in der Seele des Menschen eine archetypische Verankerung [haben] «(Jung, 2010, Klappentext). Damit sind diese archetypischen Erfahrungen allen Menschen und Kulturen gemein und überdauern historische und gesellschaftliche Entwicklungen. Als Beispiele hierfür seien zwei Fallbeispiele Jung'scher Psychoanalytiker angeführt, in denen im therapeutischen Prozess auf archetypische Deutungen zurückgegriffen wurde:

Roesler berichtet von einem Patienten,

»eine $[\mathrm{m}]$ jungen Mann im Studium, der eine spätadoleszente Problematik hatte, also Schwierigkeiten mit dem Übergang zum psychischen Erwachsenwerden. Er berichtete einen Traum, in dem von anderen, heilig erscheinenden Personen eine Art Ritual an ihm vollzogen wurde. Seine Beschreibung entsprach bis ins Detail einem Initiationsritus, wie er bei einigen Stämmen Ostafrikas praktiziert wird « (Roesler, 2009, S. 268).

Roesler weist weiter darauf hin, dass nicht auszuschließen sei, dass der Patient auf anderem Wege von diesem Ritus erfahren habe. Dennoch erkennt er eine »erstaunliche[e] und erklärungsbedürftig[e] « (ebd.) Übereinstimmung zwischen dem ostafrikanischen Initiationsritus und der individuellen Psychodynamik des Patienten, bei der ebenfalls die archetypische Initiation ins Erwachsenleben im Mittelpunkt stand.

Wolfram Gekeler (2012) identifiziert im therapeutischen Sandbild eines neunjährigen Patienten mit türkisch-kurdisch-deutschen Zugehörigkeiten ebenfalls archetypische Elemente, die über die individuelle Familiengeschichte des Patienten und über konkrete Erlebnisse in den gesellschaftlichen Kontexten Türkei, Kurdistan und Deutschland hinausgehen. Stattdessen gehe es »um Urahnen, Welteltern, somit auch um ein sehr tiefes Heimatgefühl, das er jenseits der Schwierigkeiten mit der kulturellen Identität finden konnte « (ebd., S. 355):

»So stellte B. [der Patient; I. O.-T.] etwas dar, was ich in einem Navajo-Schöpfungsmythos, ebenfalls in einem (indianischen, gestreuten) Sandbild, wiedergefunden habe: Vater und Mutter, die das Leben in ihren Kindern weitergeben, finden ihre Entsprechung in der kosmischen Dimension als Himmel und Erde. Vater Himmel wird mit den Symbolen Sonne, Mond und Milchstraße (Sterne) dargestellt, Mutter Erde mit Pflanzen. Es sind weitere Elemente des Weiblichen und Männlichen in B.s Bild zu finden: Frauen schöpfen Wasser, der Turm bietet Ausblick über das belebte Meer, am Strand ist die Grenze zwischen Erde und Wasser zu erleben - ein Bild für die transzendente Funktion. Der betende Mann stellt geistige Aspekte dar, in einer Haltung, die an die Begegnung des Einzelnen mit Gott im Islam erinnert. (Im Zoroastrismus, der ursprünglichen Religion der Kurden, stellen Sonne, Mond und Sterne die >Kerzen Gottes< dar - ein weiterer religiöser Aspekt) « (Roesler, 2009, S. 354). 
Eine Psychotherapie in der (Post-)Migrationsgesellschaft lässt sich nun in ihrer Ausrichtung meines Erachtens genau in dem Spannungsverhältnis verorten, das auch in den Beispielen sichtbar wird: zwischen gemeinsamem archetypischen Erleben qua Menschsein sowie als Teil einer postmigrantischen Gesellschaft und einem individuellen und subjektiven Erleben archetypisch angelegter Strukturen und daraus hervorgehender Bilder. Bedeutsam und gleichzeitig vernachlässigt sind dabei jedoch auch Positionierungen in den Machtverhältnissen der Migrationsgesellschaft und daraus resultierende persönliche Erfahrungen und Sichtweisen, die aus rassismuskritischer Perspektive in den Mittelpunkt rücken und nicht zuletzt für den Therapieprozess von Bedeutung sind (Oberzaucher-Tölke, 2017).

\subsection{Kritische Revision}

Trotz (oder gerade wegen!) ihres überpersönlichen, generationen- und kulturenübergreifenden Anspruchs muss eine psychologische Theorie wie die Analytische Psychologie, die vor mehr als 100 Jahren entstand, auch in ihrem Entstehungskontext gelesen und interpretiert werden. Abgesehen von der Weiterentwicklung psychoanalytischer Theorien im Allgemeinen (hierzu exemplarisch Mertens, 2014) wäre Jungs Theorie aus rassismuskritischer und postmigrantischer Perspektive ansonsten von vorneherein für eine zeitgemäße Psychotherapie in der Migrationsgesellschaft disqualifiziert: So sind seine Nord- und Ostafrikareisen seit den 1920er Jahren, wie sie unter anderen Gerhard Wehr (2009) in einer Biografie Jungs beschreibt, eindeutig im Kontext des europäischen Kolonialismus zu sehen. Dieser war bekanntermaßen geprägt durch Fremdherrschaft, Verdrängung und Ausbeutung vonseiten der kolonialisierenden Europäer und basierte auf rassistischen Annahmen und Argumentationsweisen, die z. B. allein Weißen geistige Fähigkeiten zuschrieben, Asiaten die Betriebsamkeit absprachen und Schwarze auf ihre Körperlichkeit reduzierten (Kerner, 2012). Dass auch Jung ein Kind dieser Epoche und damit nicht zuletzt in rassistische Diskurse verstrickt war, wird unter anderem in seinen Aussagen zur arabischen Kultur deutlich, dessen Angehörige er als » aus Affekten lebende, nicht reflektierende Menschen « (Jung, zit.n. Wehr, 2009, S. 197) bezeichnet, genauso wie er im »Primitiven« der Ostafrikaner im Verlauf seiner Reise eine »Gefahr « für sich sieht (Jung, zit.n. Wehr, 2009, S. 215). Weiterhin sind an dieser Stelle Jungs Affirmationen der Ideologie des Nationalsozialismus zu benennen, die bereits an anderen Stellen vielfach ausführlich und differenziert dargestellt wurden (z. B. Evers, 1987 sowie die Beiträge in der Zeitschrift Analytische Psychologie, Heft 168). So ging Jung unter anderem von einem spezifisch jüdischen und spezifisch arischen Unbewussten der jeweiligen »Rassen « aus, wobei »das arische Unbewusste [...] ein höheres Potential als das jüdische« habe (Jung, zit.n. Wehr, 2009, S. 278). Es soll bei 
der Auswahl und Zitation dieser Aussagen nicht darum gehen, Jung als Rassisten zu entlarven. Vielmehr soll begründet werden, warum seine Theorien, die in einem bestimmten gesellschaftlich-historischen Kontext entstanden, aus heutigen Perspektiven auf Migration und Rassismus einer kritischen Revision und Überarbeitungen bedürfen. Bei der nun anschließenden Revision der Archetypenlehre beziehe ich mich vor allem auf ihre diskursive Beschaffenheit, den ihr impliziten Biologismus sowie auf postmigrantische Potenziale einer Jung'schen Psychotherapie. Da das Archetypenkonzept nicht als konsistente Theorie gelten kann, sondern unter anderem von erkenntnistheoretischen Widersprüchen durchzogen ist (Roesler \& Sotirova-Kohli, 2013), kann im Rahmen dieser Revision nur schlaglichtartig auf einzelne Aspekte des Konzepts eingegangen werden.

Die Archetypenlehre C. G. Jungs weist grundsätzlich einen apriorischen Charakter auf (Vogel, 2012), worauf unter anderen die Aussage Jungs hinweist, das kollektive Unbewusste sei präexistent und nur in sekundärer Form, z. B. in Form von archetypischen Bildern erfahrbar (Jung, 1995 [1936b, \$90). Zunächst ist die Archetypenlehre damit in konzeptioneller Hinsicht durchaus anschlussfähig an den Foucault'schen Diskursbegriff, nach dem auch Diskurse »der Verfügbarkeit und dem unmittelbaren Zugriff denkender, sprechender und handelnder Subjekte entzogen bleiben und diesen vorgängig sind « (Bublitz, 2003, S. 6). Nicht umsonst werden von Diskurstheoretiker_innen Analogien zur Psychoanalyse hergestellt, indem die Infragestellung der Souveränität und Rationalität des Subjekts als Gemeinsamkeit identifiziert wird und epistemische Strukturen des Diskurses als » positives Unbewusstes « und damit » als Existenz eines gegebenen Wissens zu einer bestimmten Zeit, in einer bestimmten Gesellschaft « (ebd., S. 27) definiert werden. Auch Jungs Aussage, es handle sich bei Archetypen »nicht um vererbte Vorstellungen, sondern um ererbte Möglichkeiten von Vorstellungen « (Jung, 1995 [1936b], §135), ist durchaus kompatibel mit dem Foucault’schen Grundsatz, das Subjekt sei immer nur im Rahmen von es konstituierenden Diskursen möglich und denkbar. In konzeptioneller Hinsicht weisen also die Archetypenlehre und die Foucault’sche Diskurstheorie grundsätzlich Gemeinsamkeiten auf, nämlich hinsichtlich der Annahme einer latenten »Vorwirklichkeit «, die das Erleben von Subjekten strukturiert und damit in gewisser Weise determiniert. Der Unterschied besteht allerdings darin, dass Jung hinsichtlich der Archetypen von einem angeborenen Phänomen ausgeht, während Diskurse als durch ihre Akteure hervorgebracht und damit als gemacht zu verstehen sind. Dadurch besteht gleichzeitig die Möglichkeit ihrer Dekonstruktion, während sich den Archetypen im menschlichen Erleben in gewisser Weise nicht »entkommen « lässt. Wird allerdings auch die Archetypenlehre selbst als Diskurs verstanden und sie damit als Gegenstand einer kritischen Diskursanalyse in den Blick genommen, so ergibt sich Folgendes: Zunächst geht es grundsätzlich darum, den Zusammenhang von Wahrheit (einer wissenschaftlichen Theorie) und Macht zu erforschen, also »die gesellschaft- 
lichen Ideale und Weltbilder, die sich bis in die Alltagsethiken und -milieus, bis in die Subjektivität hinein fortsetzen, als historisch gewordene zu dekonstruieren und sie damit als verbindliche Vorschriften außer Kraft zu setzen « (Bublitz, 2003, S. 40). Denn: »Was sich an Lehrmeinungen und Idealen durchsetzt, ist nicht die Kraft eines übergreifenden Sinns. Die Setzung und Veränderung der Werte geschieht vielmehr im Verhältnis zum Machtzuwachs derer, die sich im Kräfteverhältnis durchsetzen « (ebd., S. 42f.). Die Archetypenlehre ist damit als ein (wissenschaftlicher) Diskurs über den Menschen und seine Psyche in den Blick zu nehmen, der sich im Rahmen von bestimmten Kräfteverhältnissen durchgesetzt hat und gleichsam als Wahrheit funktioniert. Gleichzeitig ist die Archetypenlehre wiederum auch als eine Art Gegendiskurs zum dominant(er)en psychologischen Diskurs der ausschließlichen Existenz eines persönlichen Unbewussten nach Freud zu analysieren, dem im psychoanalytischen Kräfteverhältnis sicherlich eine machtvollere Position zugeschrieben werden kann als dem Jung'schen Diskurs des kollektiven Unbewussten.

Was bedeutet dies nun für die postmigrantische Perspektive auf die Archetypenlehre? Grundsätzlich sind »die gesellschaftlichen Bedingungen wissenschaftlicher Erkenntnis im Blick [zu] behalten « (Messerschmidt, 2011, S. 82). Vor allem bei der (wissenschaftlichen) Thematisierung von Migration herrschen, auch in einer postmigrantischen Gesellschaft, jedoch noch immer asymmetrische Beziehungen zwischen den Thematisierenden und den Thematisierten vor. Dies macht eine gesellschaftliche und auch historische Standortbestimmung der Thematisierenden besonders erforderlich. Bei C. G. Jungs Position handelt es sich um die Perspektive eines privilegierten, weißen, männlichen Schweizers im Kontext einer Zeit, die von Kolonialismus und Nationalsozialismus und damit dem Selbstverständnis einer »höherwertigen weißen Rasse « geprägt war (s. o.). Von diesem gesellschaftlichen Standpunkt heraus eine psychologische Theorie mit dem Anspruch globaler und universeller Gültigkeit zu formulieren, ist durchaus kritisch zu sehen, zumal die Diskursanalyse und damit auch rassismuskritische und postmigrantische Perspektiven jeder Form globaler Theoriebildung grundsätzlich eine Absage erteilen (Bublitz, 2003). Der westlichen psychologischen Theorien mit universellem Anspruch inhärente Eurozentrismus wurde dementsprechend in den letzten, post-jungianischen Jahrzehnten ausführlich diskutiert und (macht-)kritisch reflektiert, z. B. im Rahmen der Ethnopsychoanalyse (siehe zur Einführung Reichmayr, 2003) sowie in postkolonialen Ausführungen zur Psychoanalyse (z. B. Said, 2004).

Eine weitere zu überdenkende Grundlage der Jung'schen Archetypenlehre ist ihr impliziter Biologismus. Durch die Annahme, archetypische Strukturen wie der Mutter- und Vaterkomplex seien grundsätzlich angeboren, verschob C. G. Jung »komplexe intergenerationelle und soziokulturelle Vorgänge in den Bereich biologischer Instinktpositionen « (Mertens, 2014, S. 84). Dies wurde auch von Jung'schen Psychoanalytiker_innen selbst infrage gestellt und anhand aktueller wissenschaftlicher Erkenntnisse 
revidiert (z. B. Roesler, 2009). Interessanterweise hat sich auch Jung selbst von biologi(sti)schen Grundlagen der Psychologie distanziert, indem er die Psychologie als »weder Biologie noch Physiologie noch irgendeine andere Wissenschaft als eben das Wissen um die Seele « definiert (Jung, 1995 [1934], \$63). Aus rassismuskritischer Perspektive sind nun Biologismen in der Psychologie besonders brisant, da die Annahme biologischer Unterschiede seit jeher die Grundlage für jede Form von Rassismus darstellt. Dies zeigt sich nicht zuletzt an Jungs Unterscheidung von jüdischer und nicht jüdischer Psychologie und von einem je spezifischen jüdischen und »arischen « kollektiven Unbewussten. Heute sind im Unterschied dazu Unterscheidungspraxen, die sich auf das biologische Merkmal »Rasse « beziehen, kaum mehr gesellschaftsfähig. Wie der französische Philosoph Etienne Balibar herausgearbeitet hat, werden diese Unterscheidungen jedoch durch einen Neo-Rassismus bzw. Kultur-Rassismus ersetzt. Im Zuge der offiziellen Entkolonialisierung habe sich dieser entwickelt als ein Rassismus, »dessen vorherrschendes Thema nicht mehr die biologische Vererbung, sondern die Unaufhebkarkeit der kulturellen Differenzen ist « (Balibar, 1990, S. 28). Das Unterscheidungsmerkmal »Rasse « sei dabei durch »Kultur « abgelöst worden, um den Fortbestand von Rassismus als Macht- und Herrschaftsverhältnis quasi unter neuem Namen zu sichern. Dies wird nun an der Stelle relevant, wo »kulturspezifische Ausprägungen « bzw. »Überformungen « von Archetypen hervorgehoben werden (z. B. Güç, 2006; Kast, 2007; siehe 4.3) und dabei ein statischer und deterministischer Kulturbegriff zugrunde liegt. Hier ist eine Biologisierung kultureller Unterschiede und ihrer Folgen zumindest mitzudenken und in ihren möglichen Effekten zu reflektieren.

Die kritische Revision der Archetypenlehre (vorerst) abschließend, geht es nun aus postmigrantischer Perspektive darum, »Strukturen, Institutionen und politische Kulturen nachholend (also postmigrantisch) an die erkannte Migrationsrealität [anzupassen] « (Foroutan, 2015). Dies gilt damit auch für den Bereich der Psychotherapie als Bereich der öffentlichen Gesundheitsversorgung im Allgemeinen sowie für die Jung'sche Analytische Psychologie im Besonderen. Ihre Konzepte und (impliziten) Annahmen müssen der Migrationsrealität in Deutschland angepasst werden, um zukunftsfähig zu bleiben und nicht zu einer Psychotherapie der Etablierten zu werden. So kann z. B. die therapeutische Deutung archetypischer Symbole in der Therapie und im eigenen Entwicklungsprozess in ihren Voraussetzungen und Vorannahmen kritisch hinterfragt werden und damit der therapeutische Blick letztlich weiter und offener werden.

\subsection{Potenziale für eine postmigrantische Psychotherapie}

Welche Potenziale birgt nun die Archetypenlehre für eine an die Migrationsrealität angepasste und damit postmigrantische Psychotherapie? Zur Beantwortung dieser Frage 
soll zunächst noch einmal am universellen Anspruch des Archetypenkonzepts angesetzt werden sowie schließlich an seiner Vieldeutigkeit und dem daraus resultierenden schöpferischen Potenzial.

Durch die Annahme eines allen Menschen gemeinsamen Strukturmusters menschlicher Erfahrung liefert die Archetypenlehre zunächst so etwas wie eine gemeinsame Basis für eine Psychotherapie in der postmigrantischen Gesellschaft. Diese gilt dann qua Menschsein und nicht aufgrund einer angenommenen »kulturellen Gleichartigkeit «. Aus einer Perspektive, die Kulturalisierungen und Ethnisierungen menschlichen Verhaltens und Erlebens grundsätzlich kritisch gegenübersteht, birgt diese Annahme das Potenzial, in der Therapie statt von (kulturellen) Differenzen zunächst einmal von einem gemeinsamen Erleben und ähnlichen, kollektiven Erfahrungen auszugehen, z. B. Kind-von-Eltern-Sein statt Kind-von-Migranteneltern-Sein. Dieses gemeinsame Erleben gilt dann auch für vermeintlich migrationsspezifisch anmutende Themen wie z. B. Fremdheit, Heimat oder die Suche nach Zugehörigkeit(en). Auch das Konzept einer postmigrantischen Gesellschaft als gemeinsamer Lebens- und Erfahrungsort aller ihrer Mitglieder (also auch der vermeintlich Nicht-Migrierten) kann, wie das kollektive Unbewusste und die Archetypen, als eine gemeinsame Basis funktionieren. Ist hingegen von »Patient_innen mit Migrationshintergrund « die Rede, als handele es sich dabei um eine eigene Spezies, so werden die Patient_innen (1.) schon allein über die Benennungspraxis zu »Anderen « gemacht und ihnen damit Zugehörigkeit sowie ein gemeinsamer Erfahrungshintergrund abgesprochen. Für die Therapie kann dies (2.) bedeuten, dass über den $\gg$ Migrationshintergrund $\ll$ bestimmte zu bearbeitende Themen (z. B. Identitätskrisen durch »kulturelle Zerrissenheit «; Oberzaucher-Tölke, i. E.) vorausgesetzt und den Patient_innen damit quasi aufgedrängt werden. Gleichzeitig ist es ein Privileg mehrheitsangehöriger Therapeut_innen, Hautfarbe und andere Merkmale (Name, Kopftuch, Sprache), die einen Patienten oder eine Patientin vor dem Hintergrund rassistischen und postkolonialen Wissens als $\gg$ Anderen « markieren, als $\gg$ nicht bedeutsam « wahrzunehmen (Oberzaucher-Tölke, 2017). Für das Gegenüber sind sie nämlich meist sehr wohl relevant, unter anderem als Ursache für Rassismus- und Diskriminierungserfahrungen. Wird also der sogenannte »Migrationshintergrund « seitens des Therapeuten völlig ausgeblendet, so besteht die Gefahr der Dethematisierung spezifischer Erfahrungen von Ungleichbehandlung. Es gilt also, in der postmigrantischen Therapie nicht zuletzt auf der Beziehungsebene dieses Spannungsverhältnis von Gemeinsamkeiten und Unterschieden anzuerkennen und auszuhalten, wobei unter anderem die psychoanalytischen Konzepte von Containing ${ }^{3}$ und Holding ${ }^{4}$ hilfreich sein können.

Ein weiteres postmigrantisches Potenzial der Archetypenlehre sehe ich in ihrem sowohl vieldeutigen als auch schöpferischen Charakter, wie ihn unter anderen die Psychoanalytikerin Verena Kast (2007) beschreibt. So sind archetypische Symbole nach Jung 
» vieldeutig, ahnungsreich und im letzten Grund unausschöpfbar « (Jung, 1995 [1934], $\$ 80$ ), was eine persönliche Freiheit in ihrer Ausgestaltung mit sich bringt. Archetypische Vorstellungen und Bilder müssen nach Kast gestaltet und in die Sprache der Gegenwart übersetzt werden, wenn sie nicht, so Jung selbst, » wegen zunehmender Altertümlichkeit ihres Begriffes ihre Bannkraft einbüßen [sollen] (Jung, zit.n. Kast, 2007, S. 27). Wird nun davon ausgegangen, archetypische Bilder seien kulturell überformt (Kast, 2007), und sich dabei implizit auf einen klassischen Kulturbegriff bezogen, besteht die Gefahr der bereits erwähnten » monokulturellen Zwangsjacke «. Diese kommt im Therapieprozess konkret dann zum Tragen, wenn mehrheitsangehörige Therapeut_innen vorschnell vermeintlich »kulturell andere Symbole « bei »Patient_innen mit Migrationshintergrund « erkennen, z.B. im Kontext der Arbeit mit den therapeutischen Methoden des Sandspiels, des Malens oder der Traumdeutung. Aus postmigrantischer Perspektive müssen stattdessen im Therapiekontext vor allem neue, hybride Bilder und Symbole Raum bekommen, die einem (Er-)Leben in der Postmigrationsgesellschaft entsprechen. Für die Patient_innen geht es also darum, sich ihr »Gewand selber zu nähen « (Jung,1995 [1934], \$24), ohne auf von kulturalistischen Diskursen geprägte, monokulturelle Symbole festgelegt zu werden. Hier kommt nun der schöpferische Mensch ins Spiel, der sich beim Nähen seines ganz eigenen Gewandes vom »schöpferischen Drang in allem Lebendigen « (Kast, 2007, S. 29) leiten lassen dürfen muss - auch auf neue, vielleicht hybride Wege abseits von Diskursen um eigene und fremde Kultur(en). Dazu heißt es im Roman Restaurant Dalmatia (2013) der als postmigrantisch bezeichneten Autorin Jagoda Marinic: »Man braucht viel Kraft, um aus alten Stoffen neue Kleider zu nähen « - möge Psychotherapie diese Kraft zu stärken wissen, anstatt sie in monokulturellen Zwangsjacken zu ersticken.

Nicht zuletzt möchte ich also für ein neues, postmigrantisch informiertes und konsequent anzuwendendes Verständnis von Kultur in der Psychotherapie plädieren. Als Diskussionsgrundlage können hier transkulturelle Ansätze dienen, die sich auf einen konstruktivistischen, durch Hybridität gekennzeichneten Kulturbegriff stützen (z. B. Nadig, 2006). Leider wird bisher in entsprechend betitelten Konzeptionen oft doch wieder auf klassische Kulturkonzepte zurückgegriffen (vgl. Oberzaucher-Tölke, 2018) - wahrscheinlich deshalb, weil der Ansatz der Transkulturalität in seiner Komplexität methodisch oft nicht leicht zu fassen ist, wie die Ethnopsychoanalytikerin Maya Nadig (2006, S. 69) selbst anmerkt. Schließen möchte ich also mit einem Zitat C.G. Jungs: »Dass man diesen ewigen Bildern erliegt, ist eine an sich normale Sache« (Jung, 1995 [1934], \$11). Ja, es ist aus Jung'scher Perspektive eine normale Sache, ewigen Bildern zu erliegen, weil sie archetypisch sind. Es ist auch eine normale Sache, »ewigen «, oft stereotypen und vereinfachenden Bildern über den »Anderen « und »seine Kultur « zu erliegen, weil Diskurse über Kultur und Fremdheit im Zusammenhang mit Migration unsere Wahrnehmungen und Identitäten bilden und prägen. 


\section{Gleichzeitig sind viele dieser ewigen Bilder aus einer postmigrantischen Perspektive de- finitiv auch » ewig gestrig « und wollen neu erschaffen werden.}

\section{Anmerkungen}

1 Der Begriff natio-ethno-kulturell thematisiert die Überbestimmung, den diffusen Charakter sowie die Unschärfe von Unterscheidungen wie »deutsch«, »türkisch«, »italienisch« und »arabisch«, die dennoch auf politischer, sozialer und wissenschaftlicher Ebene alltäglich und wirksam sind (Mecheril, 2003).

2 Angelehnt an Mecheril (2004, Kap. 6), der sich selbst gegenüber dem »Postmigrantischen« allerdings kritisch äußert (siehe auch Mecheril, 2014).

3 Aufnehmen von unverarbeiteten und damit zunächst unerträglichen Gefühlen eines Patienten durch den Psychoanalytiker, um diese stellvertretend für den Patienten zu verarbeiten (nach Wilfred Bion).

4 Ermöglichung einer therapeutischen Beziehung, die in Anlehnung an die frühe Mutter-KindBeziehung durch Zuverlässigkeit sowie haltgebende und realitätsbewahrende Faktoren gekennzeichnet ist (nach Donald Winnicott).

\section{Literatur}

Balibar, É. (1990). Gibt es einen »Neo-Rassismus«? In É. Balibar \& I. Wallerstein, Rasse, Klasse, Nation. Ambivalente Identitäten (S. 23-38). Hamburg: Argument.

Broden, A. \& Mecheril, P. (2007). Migrationsgesellschaftliche Re-Präsentationen. Eine Einführung. In A. Broden \& P. Mecheril (Hrsg.), Re-Präsentationen. Dynamiken der Migrationsgesellschaft (S. 7-28). Düsseldorf: IDA-NRW.

Bublitz, H. (2003). Diskurs. Bielefeld: Transcript.

Castro Varela, M. \& Dhawan, N. (2005). Postkoloniale Theorie. Eine kritische Einführung (2. Aufl.). Bielefeld: transcript.

Diehm, I. \& Radtke, F.-O. (1999). Erziehung und Migration. Stuttgart: Kohlhammer.

Erman, M. (2014). Der Andere in der Psychoanalyse. Die intersubjektive Wende. Stuttgart: Kohlhammer. Evers, T. (1987). Mythos und Emanzipation. Eine kritische Annäherung an C. G. Jung. Hamburg: Junius.

Foroutan, N. (2015). Die Einheit der Verschiedenen. Integration in der postmigrantischen Gesellschaft. http:// www.bpb.de/gesellschaft/migration/kurzdossiers/205183/integration-in-der -postmigrantischen-gesellschaft (02.11.2016).

Gekeler, W. (2012). »Es ist einfach ein fremdes Land«. Aus der Behandlung eines 9-jährigen türkisch-kurdisch-deutschen Jungen. Analytische Kinder- und Jugendlichen-Psychotherapie, 155(3), 347-359.

Güç, F. (2006). Transkulturelle Psychoanalyse. Ein Therapieansatz für Migranten. In E. Wohlfahrt \& M. Zaumseil (Hrsg.), Transkulturelle Psychiatrie - Interkulturelle Psychotherapie (S. 239-275). Heidelberg: Springer.

Hegemann, T. \& Salman, R. (2010). Transkulturelle Psychiatrie. Konzepte für die Arbeit mit Menschen aus anderen Kulturen. Köln: Psychiatrie-Verlag.

Hess, S. (2015). Politiken der Unsichtbarmachung. Eine Kritik der Wissens- und Bilderproduktion zu Migration. In E. Yildiz \& M. Hill (Hrsg.), Nach der Migration. Postmigrantische Perspektiven jenseits der Parallelgesellschaft (S. 49-64). Bielefeld: transcript. 
Jäger, S. (1993). Kritische Diskursanalyse. Eine Einführung. Duisburg: DISS.

Jung, C.G. (1995 [1934]). Über die Archetypen des kollektiven Unbewussten. Gesammelte Werke, Band 9/1 (S. 11-51). Düsseldorf: Walter-Verlag.

Jung, C.G. (1995 [1936a]). Der Begriff des kollektiven Unbewussten. Gesammelte Werke, Band 9/1 (S. 55-66.). Düsseldorf: Walter-Verlag.

Jung, C. G. (1995 [1936b]). Über den Archetypus mit besonderer Berücksichtigung des Animabegriffs. Gesammelte Werke, Band 9/1 (S. 67-87). Düsseldorf: Walter-Verlag.

Jung, C. G. (2010). Archetypen. München: dtv.

Kast, V. (2007). Die Tiefenpsychologie nach C. G. Jung. Eine praktische Orientierungshilfe. Stuttgart: Kreuz.

Keller, R. (2011). Diskursforschung. Eine Einführung für Sozialwissenschaftlerlnnen. Wiesbaden: VS.

Kerner, I. (2012). Postkoloniale Theorien zur Einführung. Hamburg: Junius Verlag.

Kimil, A., Waldhoff, H.-P. \& Salman, R. (2013). Wer versteht schon diese Familien? Ressourcen und Schwierigkeiten von MigrantInnen und ihren Familien im Kontext von Beratung und Therapie. Zeitschrift für systemische Therapie und Beratung, 31(2), 63-72.

Machleidt, W. \& Heinz, A. (Hrsg.). (2011). Praxis der interkulturellen Psychiatrie und Psychotherapie. München: Urban \& Fischer.

Marinic, J. (2013). Restaurant Dalmatia. Hamburg: Hoffmann und Campe.

Mecheril, P. (2003). Prekäre Verhältnisse. Über natio-ethno-kulturelle (Mehrfach-)Zugehörigkeit. Münster: Waxmann.

Mecheril, P. (2004). Einführung in die Migrationspädagogik. Weinheim, Basel: Beltz.

Mecheril, P. (2014). Was ist das X im Postmigrantischen? sublurban. Zeitschrift für kritische Stadtforschung, 2(3), 107-112.

Mecheril, P. \& Melter, C. (2009). Rassismustheorie und -forschung in Deutschland. Kontur eines wissenschaftlichen Feldes. In P Mecheril \& C. Melter (Hrsg.), Rassismuskritik. Band 1: Rassismustheorie und -forschung (S. 13-22). Schwalbach am Taunus: Wochenschau.

Mecheril, P., Thomas-Olalde, O., Melter, C., Arens, S. \& Romaner, E. (2013). Migrationsforschung als Kritik? Erkundung eines epistemischen Anliegens in 52 Schritten. In P. Mecheril, O. Thomas-Olalde, C. Melter, S. Arens \& E. Romaner (Hrsg.), Migrationsforschung als Kritik? Spielräume kritischer Migrationsforschung (S. 7-55). Wiesbaden: Springer VS.

Meinhardt, R. \& Schulz-Kaempf, W. (2015). Einwanderungen nach Deutschland und Migrationsdiskurse in der Bundesrepublik - eine Synopse. In R. Leiprecht \& A. Steinbach (Hrsg.), Schule in der Migrationsgesellschaft. Ein Handbuch (S. 54-92). Schwalbach am Taunus: Wochenschau Verlag.

Mertens, W. (2014). Psychoanalyse im 21. Jahrhundert. Eine Standortbestimmung. Stuttgart: Kohlhammer. Messerschmidt, A. (2011). Involviertes Forschen. Reflexionen von Selbst- und Fremdbildern. In I. Breinbauer \& G. Weiß (Hrsg.), Orte des Empirischen in der Bildungstheorie (S. 81-95). Würzburg: Königshausen \& Neumann.

Nadig, M. (2006). Transkulturelle Spannungsfelder in der Migration und ihre Erforschung. Das Konzept des Raums als methodischer Rahmen für dynamische Prozesse. In E. Wohlfahrt \& M. Zaumseil (Hrsg.), Transkulturelle Psychiatrie - Interkulturelle Psychotherapie (S. 67-80). Heidelberg: Springer.

Oberzaucher-Tölke, I. (2013). »Deine Kultur, meine Kultur«: Warum es sich lohnt, die Kulturbrille hin und wieder abzusetzen. http://www.kindergartenpaedagogik.de/2271.html (02.11.2016).

Oberzaucher-Tölke, I. (2014). »Der andere Patient« - Die Konstruktion kultureller Differenz in der Psychotherapie aus rassismuskritischer Perspektive. Psychologie \& Gesellschaftskritik, 150(2), 91-102.

Oberzaucher-Tölke, I. (2017). Wer ist hier »fremd«? Migration und Fremdheit in Psychoanalyse und Psychotherapie. Analytische Psychologie, 187, 84-104.

Oberzaucher-Tölke, I. (2018). Beratung und Therapie in der Migrationsgesellschaft. In B. Blank, S. Gögercin, K. Sauer \& B. Schramkowski (Hrsg.), Soziale Arbeit in der Migrationsgesellschaft. Grundlagen - Konzepte-Handlungsfelder (S. 657-666). Wiesbaden: Springer VS. 
Oberzaucher-Tölke, I. (i. E.). Die Behandlung der sAnderen«. Othering in der Psychoanalyse von Kindern und Jugendlichen in der Migrationsgesellschaft. In C. Hunner-Kreisel \& J. Wetzel (Hrsg.), Rassismuskritik und Soziale Arbeit, Sonderheft neue praxis.

Reichmayr, J. (2003). Ethnopsychoanalyse. Geschichte, Konzepte, Anwendungen. Gießen: PsychosozialVerlag.

Roesler, C. (2009). Archetypen - sozial, nicht biologisch. Eine Reformulierung der Archetypentheorie auf Grundlage neuer Erkenntnisse aus Neurowissenschaften, Humangenetik, Entwicklungs- und Kulturpsychologie. Analytische Psychologie, 40(3), 276-303.

Roesler, C. \& Sotirova-Kohli, M. (2013). Das psychische Erbe der Menschheit. Forschungsstand und empirische Studien zum Archetypenkonzept C. G. Jungs. Forum Psychoanalyse, 30, 133-155.

Römhild, R. (2014). Diversität?! Postethnische Perspektiven für eine reflexive Migrationsforschung. In B. Nieswand \& H. Drotbohm (Hrsg.), Kultur, Gesellschaft, Migration. Studien zur Migrations- und Integrationspolitik (S. 255 -270). Wiesbaden: Springer.

Römhild, R. (2015). Jenseits ethnischer Grenzen. Für eine postmigrantische Kultur- und Gesellschaftsordnung. In E. Yildiz \& M. Hill (Hrsg.), Nach der Migration. Postmigrantische Perspektiven jenseits der Parallelgesellschaft (S. 37-48). Bielefeld: transcript.

Said, E. (2004). Freud und das Nicht-Europäische. Zürich: Dörlemann.

Schnocks, D. (2013). Mit C. G. Jung sich selbst verstehen. Stuttgart: Kohlhammer.

Vogel, R. (2012). Analytische Psychologie und die ihr angemessenen Forschungsmethoden. Epistemologische Überlegungen zu ihrem Status als Wissenschaft. Analytische Psychologie, 43(167), 74-105.

Wehr, G. (2009). Carl Gustav Jung. Leben - Werk - Wirkung. München: Telesma.

Wohlfahrt, E. \& Zaumseil, M. (Hrsg.). (2006). Transkulturelle Psychiatrie - Interkulturelle Psychotherapie. Interdisziplinäre Theorie und Praxis. Heidelberg: Springer.

Yildiz, E. (2015). Postmigrantische Perspektiven. Aufbruch in eine neue Geschichtlichkeit. In E. Yildiz \& M. Hill (Hrsg.), Nach der Migration. Postmigrantische Perspektiven jenseits der Parallelgesellschaft (S. 19-36). Bielefeld: transcript.

Yildiz, E. \& Hill, M. (2015). Einleitung. In E. Yildiz \& M. Hill (Hrsg.), Nach der Migration. Postmigrantische Perspektiven jenseits der Parallelgesellschaft (S. 9-16). Bielefeld: transcript.

\section{Die Autorin}

Inga Oberzaucher-Tölke, Dr. phil., Dipl.-Päd., befindet sich in Ausbildung zur Analytischen Kinder- und Jugendlichenpsychotherapeutin am C. G. Jung-Institut Stuttgart. Ihre Arbeitsschwerpunkte sind: Rassismustheorie und -forschung, Psychotherapie und Migration, Psychoanalyse und Diskursanalyse. Kontakt: inga.oberzaucher@posteo.de 